\title{
In silico analysis of wild-type and mutant KRAS
}

\author{
Frengki $^{1,2}$, Dedi Prima Putra ${ }^{3}$, Fatma Sriwahyuni ${ }^{3}$, Daan Khambri ${ }^{1,}$ Henni Vanda $^{2}$ \\ ${ }^{1}$ Biomedicine Faculty of Medicine of Andalas University, Padang, Indonesia \\ Jl. Perintis Kemerdekaan No.94 PO BOX 49 Padang 25127 \\ ${ }^{2}$ Faculty of Veterinary Medicine of Syiah Kuala University, Banda Aceh, Indonesia \\ Jl. Tgk. Hasan Krueng Kalee No.4, Kopelma Darussalam, Syiah Kuala, Kota Banda Aceh 23111 \\ ${ }^{3}$ Faculty of Pharmacy Andalas University, Padang, Indonesia 25163 \\ Jl. Limau Manis, Limau Manis, Pauh, Kota Padang, Sumatera Barat
}

\begin{abstract}
The mutations of the KRAS gene at codons 12,13 , and 61 have been widely reported with different prognosis. In silico is one approach to explain the characteristics of the mutant genes. This study aimed to reveal the potential energy and fluctuations of the binding site and active site of wildtype KRAS (KRAS Wt) and mutant KRAS (KRAS Mt) at codons 12, 13, and 61. The samples used in this study were the sequences of KRAS Wt and KRAS Mt genes, which were subjected to in-silico analysis that included molecular homology, docking, and dynamics using MOE, PyMOL, and online CABS servers. The results showed that fluctuations in the binding site of all KRAS Mt were lower than that of KRAS Wt. On the contrary, the active site (switch I and switch II) of KRAS Mt fluctuated more widely than KRAS Wt. The potential energy of KRAS Mt before forming a complex with GTP was higher $(\mathrm{p}<0.01)$ than KRAS Wt. After this formation, it remained higher at codons 12 and 61 but lower at codons 11 and 13 ( $\mathrm{p}<0.001$ ). Mt G12A did not show any changes. The higher fluctuations in the switch I and switch II regions and the post energy of KRAS-GTP complexes may explain why types of cancers with mutations at codons 11 and 13 have a better prognosis than those with mutations at codons 12 and 61.
\end{abstract}

Keywords: fluctuations, in silico, KRAS, polymorphism, potential energy

*Corresponding author:

Frengki

Universitas Syiah Kuala

Darussalam, Banda Aceh

Jl. Tgk. Hasan Krueng Kalee No.4, Kopelma Darussalam, Syiah Kuala, Kota Banda Aceh 23111

Email: frengki_fkh@unsyiah.ac.id 


\section{INTRODUCTION}

Abnormalities in cell division are often associated with deviations of proteins involved in cell proliferation and differentiation, which are observable from any conformational changes in the geometric structure.

The Ras Protein and its family, including KRAS, NRAS, and HRAS, are proto-oncogenes, which play roles in transmitting surface receptor signals to effector protein through the PIK3/AKT and RAF/MEK/ERK pathways. These two pathways are responsible for the process of cell proliferation and differentiation. KRAS, NRAS, and HRAS can turn into oncogenes due to mutations in one or more of their base pairs that may increase cell proliferation and differentiation activities and, thereby, trigger resistance to epidermal growth factor receptor (EGFR) monoclonal antibodies (Knickelbein and Zhang, 2014).

Point mutations in the KRAS gene are a type of mutation most commonly found in cancer patients (Karnoub and Weinberg, 2008). The most frequently reported types of cancer that have this gene mutation include pancreatic cancer (up to 70-80\%) (Stephen et al., 2014; Garcea et al., 2005), colorectal cancer (up to $40 \%-90 \%$ ), lung cancer (30\%), breasts cancer $(<5 \%)$, and fewer types of cancer (de Roock et al., 2010; Forbes et al., 2009). Most of the KRAS gene mutations are localized at codons 12, 13, and 61. However, Miyakura et al. (2002) have successfully identified mutation at codon 22, and Hongyo et al. (1995) have reported one at codon 11.

Mutations at codon 12 have been reported in up to $80 \%$ of all occurrences, while mutations at codon 13 reach $17 \%$ and the rest occurs at other codons (Forbes et al., 2006). The KRAS gene mutations in pancreatic and colon cancer have been indicated at codon 12, changing the production of amino acid, that is, the substitution of cysteine for glycine (G12C) (Forbes et al., 2011). In patients with lung cancer, the mutations occur at the same codon as pancreatic and colon cancer, but the alterations in amino acid production involve the conversion of glycine to aspartate (G12D) (Stephen et al., 2014). Also, mutations have been identified at codon 13, i.e., where glycine changes to aspartate, and some other amino acids, and at codon 61 that influences the conversion of glycine to histidine. These mutations often take place in the GDP/GTP bond or called the GTPase domain.

The activities of the GTPase in KRAS Wt and KRAS Mt (G12C, G12D, G12R, G12V, G13D, $\mathrm{Q} 61 \mathrm{H}$ and $\mathrm{Q} 61 \mathrm{H}$ ) have been examined through in vitro analysis by Hunter et al. (2015). The results of this analysis showed that KRAS Mt-G12C had the highest GTPase activity, i.e., 72\% compared with KRAS Wt. Meanwhile, the GTPase activities occurring in the other mutant forms of KRAS were lower, namely $27.9 \%$ in KRAS Mt-G12D, $14.1 \%$ in G13D, $6.2 \%$ in G12V, $2.6 \%$ in G12R, $1.9 \%$ in G12A, 1.9\% in Q61H, and, the lowest, 1.1\% in Q12L compared with KRAS Wt.

The hydrolysis process can be described through the interactions between KRAS Wt and GTPase-activating protein (GAP) complex, specifically between the amino acids Q61 in KRAS Wt, as well as $\delta$ and $\beta$ GTP, with the amino acid R789 in GAP. The $\mathrm{NH}_{2}$ group of the amino acid R789 in GAP interacts with the CO group of KRAS Wt protein with a bond distance of $2.7 \AA$. The connection between the NH group of the amino acid R789 in GAP with $\mathrm{O}(\delta$ and $\beta$ ) atoms in phosphate resulted from hydrolyzed GTP occurs with the bond distances of 2.8 and 3.2 (§) (Scheffzek et al., 1997). Gao and Leif (2013) also illustrate that the side chain position of Y32 phenol of KRAS Wt protein shifts away from the $\delta$ - and $\beta$-phosphate of GTP, whereas R789 in GAP is closer to the $\delta$ - and $\beta$-phosphate of GTP. Y32 phenol group in KRAS Wt is within the distance of $7 \AA$ to the R789 in GAP, but it is shorter in KRAS Mt-D31N and D33N ( $\pm 4 \AA$ ). Accordingly, Y32 in KRAS Wt is assumed to play an essential role in opening the gate between this group and the GTP binding site.

According to Futatsugi and Tsuda (2001), the position and orientation of Q61 affect the ability to activate the water molecule 175 (W175). W175, located close to the $\delta$-phosphate in GTP, is considered as a requirement for the hydrolysis process of GTP to GDP. Krengel et al. (1990) also confirm this claim after observing KRAS Mt-G12R or G12V mutations. Aside from W175, Lysine 16 
has also been reported to be directly involved in the hydrolysis process of the phosphate groups of GTP to GDP when the distances between $\mathrm{N}$ atoms (Lys 16) and $\mathrm{O} 1$ atoms in the $\delta$-phosphate and $\beta$ phosphate of GTP are approximately 3 and $2 \AA$, respectively (Krengel et al., 1990). Mutations at codons 12,13, and 61 are believed to cause changes in the orientation and distance of some of these amino acids.

The in vitro test of the activity of the KRAS-GAP complex, including KRAS Wt and KRAS Mt (G12C, G12D, G12R, G12V, G13D, Q61H, and Q61H), has also been carried out by Hunter et al. (2015). Based on the test results, the GAP-catalyzed hydrolysis of GTP becomes lower when GTP binds to mutated KRAS. KRAS Mt-G12D exhibited the highest catalytic activity, namely $2.07 \%$ activity compared with KRAS Wt. The catalytic activities of the other mutant forms of KRAS varied from $0.12 \%$ to $0.74 \%$ ( $0.74 \%$ in G12A, $0.56 \%$ in G12V, $0.46 \%$ in $\mathrm{G} 12 \mathrm{C}, 0.46 \%$ in $\mathrm{G} 12 \mathrm{R}, 0.46 \%$ in $\mathrm{G} 13 \mathrm{D}, 0.28 \%$ in Q61L, and, the lowest, $0.12 \%$ in Q61H).

The intrinsic GTPase activity of KRAS and its sensitivity to GAP can also be predicted from other parameters, such as the fluctuations of residues in the GTPase and the two binding motifs in KRAS, namely Switch I and Switch II. Potential energy can also be used in completing the prediction of GTPase activity. The characteristics of these two parameters are useful in assessing the prognosis and healing expectancy of cancer therapy.

\section{MATERIALS AND METHOD \\ Materials}

In this experiment, some programs were used to analyze the mutation of codons. PyMOL v. 0.99 was applied for visualization, MOE 2007.09 for docking and homology modeling, and online CABS flex server for fast simulation of protein structure fluctuations. The data of the sequence of amino acids in KRAS enzyme (Homo Sapiens) was obtained from uniprot.org database (P01116). The KRAS protein template was downloaded from pdb.org (GDP ID: 1WQ1).

\section{Methods}

The sequences were transformed according to the mutated amino acids at codons 11, 12, 13, and 61 in FASTA format. The binding sites were identified using MOE, followed by creating KRAS Wt and KRAS Mt enzyme models (A11P, G12A, G12C, G12D, G13A, G13C, G13D, and Q61H). All models were evaluated structurally based on the root-mean-square deviation (RMSD) scores and plots of amino acid residues (Ramachandran Plots) (Petsko and Ringe, 2004). Both ligands and enzymes were prepared and optimized for their three-dimensional structures by adding hydrogen, removing water molecules, adding partial charges, and minimizing energy. Following this procedure was the docking process through the predetermined binding sites. The resulting enzyme-ligand complex was saved in .pdb format, while the docking value was in .mdb. All homology modeling and docking processes were carried out in MOE software.

\section{Data Analysis}

The docking results were evaluated by visualization in MOE and PyMol software. Afterward, the analysis continued to the simulation of molecular dynamics for all KRAS before (pre-test) and after (post-test) the formation of the ligand-receptor complex. In this step, MOE was employed to observe potential energy changes, while the CABS-dock web server was used to identify the fluctuations of the constituent amino acids of KRAS Wt/Mt. These data were analyzed statistically.

\section{RESULTS AND DISCUSSION}

This research aimed to investigate the potential energy and fluctuations of the binding site and active site of KRAS Wt and KRAS Mt. The results showed that all types of modeled KRAS Mt had 99\% structural similarity to the KRAS Wt gene with RMSD<1 $\AA$. Based on the Ramachandran plot analysis presented in Figure 1, all models are considered to have good quality. 


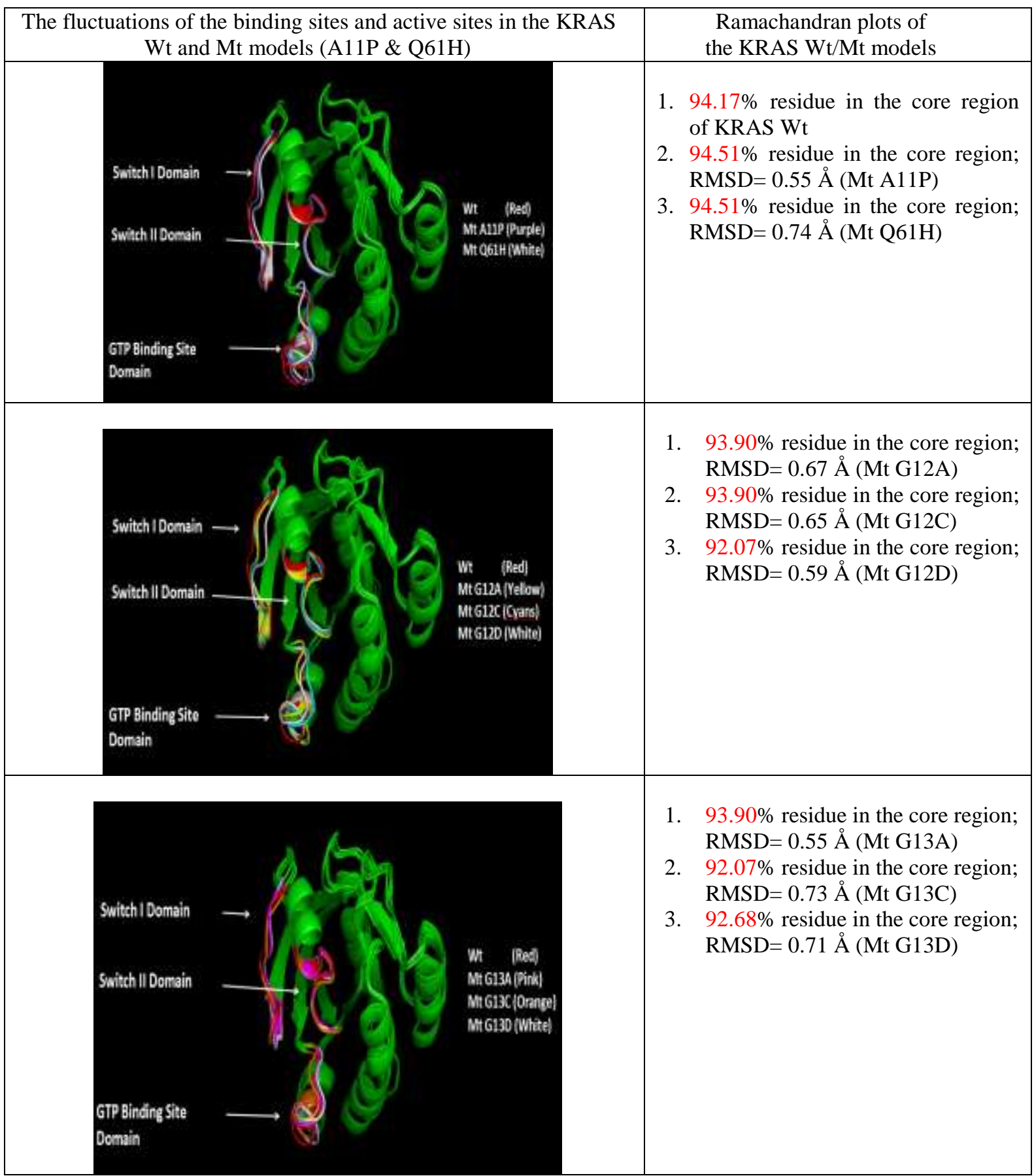

Figure 1. The fluctuations of the binding site and active site and the Ramachandran plots of the KRAS Wt/Mt models

All models in this experiment meet the requirements of a good-quality structure, namely RMSD is $<1 \AA$ and the Ramachandran plot shows $>85 \%$ of amino acid residues in the core region and $<15 \%$ 
of non-glycine residues in the disallowed region (Petsko and Ringe, 2004). Therefore, all models were subjected to the subsequent analysis.

The KRAS models in Figure 1 show similarities to each other, and the difference mainly lies in the sensitive areas, such as the GTP binding site (codons 11-16), the switch I region (codons 30-38), and the switch II region (codons 60-68). The atomic fluctuations of the GTP binding sites in KRAS Mt and KRAS Wt tended to decrease, as listed in Table I.

Table I. Regional fluctuations in "the Domains of the GTP Binding Sites" in KRAS Wt and Mt

\begin{tabular}{ccccccccccc}
\hline \multirow{2}{*}{$\begin{array}{c}\text { Amino } \\
\text { Acids }\end{array}$} & $\begin{array}{c}\text { KRAS } \\
\text { Wt }\end{array}$ & A11P & G12A & G12C & G12D & G13A & G13C & G13D & Q61H \\
\hline 11 & 2.2829 & 1.5422 & 2.8341 & 2.9584 & 1.8792 & 2.7353 & 2.4189 & 2.4697 & 3.1452 \\
12 & 4.1773 & 3.4073 & 3.6534 & 3.8766 & 2.5942 & 4.3199 & 4.385 & 4.6821 & 4.656 \\
13 & 4.9354 & 4.788 & 4.4967 & 4.8156 & 3.2418 & 4.181 & 3.8921 & 4.4376 & 4.3667 \\
14 & 5.6709 & 4.9264 & 5.4386 & 4.2243 & 3.8444 & 4.9389 & 5.0522 & 4.8114 & 4.9665 \\
15 & 1.8831 & 1.7213 & 1.5381 & 1.6565 & 1.3983 & 1.7077 & 1.3699 & 1.7444 & 1.5218 \\
16 & 1.037 & 0.861 & 0.8577 & 0.8916 & 0.8445 & 0.7514 & 0.7871 & 0.698 & 0.8127 \\
\hline
\end{tabular}

The decreased residual fluctuations in the GTP binding sites creates a more stable affinity with the ligands. According to Futatsugi and Tsuda (2001), the Lys 16 residue plays a crucial role in forming the KRAS-GTP complex. Table I shows that the fluctuations of the domains of the GTP binding sites in every KRAS Mt model have a downward trend. On the contrary, the changes in the Switch I region of KRAS Mt are more significant than KRAS Wt. The fluctuations of amino acid residues in Switch I have a substantial role in the affinity for and the interaction with GAP (Tyr 32). The data is provided in Table II.

Table II. The fluctuations in the Switch I regions of KRAS Wt and Mt

\begin{tabular}{cccccccccc}
\hline \multirow{2}{*}{$\begin{array}{c}\text { Amino } \\
\text { Acids }\end{array}$} & $\begin{array}{c}\text { KRAS } \\
\text { Wt }\end{array}$ & A11P & G12A & G12C & G12D & G13A & G13C & G13D & Q61H \\
\hline 30 & 12.1551 & 13.892 & 15.6219 & 14.3151 & 15.6995 & 14.201 & 16.1357 & 12.6599 & 13.1576 \\
31 & 12.2375 & 15.7626 & 16.8981 & 15.1176 & 16.462 & 14.3461 & 16.1103 & 13.8735 & 14.0005 \\
32 & 9.658 & 14.3209 & 13.6014 & 12.701 & 13.1438 & 12.3517 & 13.2726 & 12.1066 & 13.1425 \\
33 & 8.5287 & 11.5732 & 10.6984 & 9.7298 & 10.6493 & 10.5067 & 11.0009 & 10.5711 & 10.2635 \\
34 & 6.4181 & 8.4908 & 7.8231 & 7.3217 & 7.9124 & 8.2801 & 8.5336 & 7.8454 & 8.1675 \\
35 & 5.2895 & 6.3533 & 5.9763 & 5.5308 & 6.1249 & 6.1802 & 6.7285 & 6.1402 & 5.8718 \\
36 & 2.726 & 2.8175 & 3.0562 & 3.2252 & 3.0281 & 2.8167 & 3.3429 & 2.758 & 2.7221 \\
\hline
\end{tabular}

Table II presents significant fluctuations of Tyr 32 in all KRAS Mt. These fluctuations are assumed to reduce the open conformation of Tyr 32, which allows the insertion of Arginine 789 (amino acid; GAP) and its interactions with $\delta$ and $\beta$-phosphate (GTP) (Gao and Leif, 2013). 
Table III shows that the fluctuations of all amino acid residues in Switch II of KRAS Mt are more significant than KRAS Wt, except for 61 amino acid residues. Variations in these 61 residues in all models (KRAS Wt and Mt) are similar, except for KRAS Mt-G12A and Q61H. Q61H fluctuates almost two times higher than KRAS Wt, which lowers its affinity for and its stable interaction with GAP (Scheffzek et al., 1997). It also reduces the ability of KRAS Mt-Q61H to activate the water molecule 175 that plays a major role in the hydrolysis process of $\delta$-phosphate (GTP) (Krengel et al., 1990). The low interaction between KRAS Mt-Q61H and GAP decreases the catalytic activity of GAP in hydrolyzing GTP (1.9\%) (Hunter et al., 2015).

\section{Table III. The fluctuations in the Switch II regions of KRAS Wt and Mt}

\begin{tabular}{cccccccccc}
\hline $\begin{array}{c}\text { Amino } \\
\text { Acids }\end{array}$ & $\begin{array}{c}\text { KRAS } \\
\text { Wt }\end{array}$ & A11P & G12A & G12C & G12D & G13A & G13C & G13D & Q61H \\
\hline 60 & 3.1001 & 2.1412 & 2.8756 & 2.7751 & 2.3354 & 2.7159 & 2.7091 & 2.7396 & 2.8013 \\
61 & 5.2921 & 4.8806 & 6.0095 & 5.2461 & 4.9861 & 5.2799 & 5.2685 & 5.2171 & 7.0176 \\
62 & 8.6949 & 8.6693 & 9.0876 & 8.8353 & 9.4906 & 9.1431 & 9.4787 & 9.0909 & 10.1298 \\
63 & 5.7339 & 6.891 & 7.9939 & 7.8079 & 8.0632 & 7.3113 & 8.6477 & 7.474 & 9.9372 \\
64 & 4.3601 & 5.9804 & 5.3523 & 6.0744 & 7.2535 & 6.5767 & 7.5552 & 6.4304 & 6.2479 \\
65 & 3.0765 & 5.5553 & 5.3967 & 7.061 & 6.8789 & 6.1331 & 9.2252 & 6.6991 & 5.1462 \\
66 & 2.4828 & 4.3453 & 4.6005 & 5.5429 & 9.9412 & 5.4177 & 11.3556 & 8.7076 & 4.3489 \\
67 & 2.1309 & 4.2736 & 3.9437 & 5.4087 & 9.5005 & 5.4426 & 10.4178 & 8.2599 & 3.9634 \\
68 & 1.6122 & 3.3182 & 3.1118 & 3.9449 & 2.7546 & 3.1451 & 3.9649 & 2.6634 & 2.8068 \\
\hline
\end{tabular}

The differences in KRAS structures also affect the variations of the ligand-receptor affinity, as observed from the docking scores and the molecular dynamics simulations. The higher the docking score (more negative), the stronger the affinity for GTP (Hardono et al., 2013). Referring to the docking score, KRAS Wt has a relatively weaker affinity compared with KRAS Mt, except for G13C mutant. The ligand-receptor complex formed between GTP and KRAS Mt is stronger, and the signaling function of the messenger is expected to be more active than KRAS Wt. An increase in signaling function leads to uncontrolled cell growth and proliferation. KRAS gene mutation has been reported in almost 30\% of cancer cases (Karnoub and Weinberg, 2008). It has also been detected in $70-80 \%$ of pancreatic cancers, $40 \%$ of colon cancers, and up to $30 \%$ of lung cancers (Stephen et al., 2014; Garcea et al., 2005; De Roock et al., 2010; Forbes et al., 2009). KRAS gene mutation is associated with improved signaling function of the messenger. 


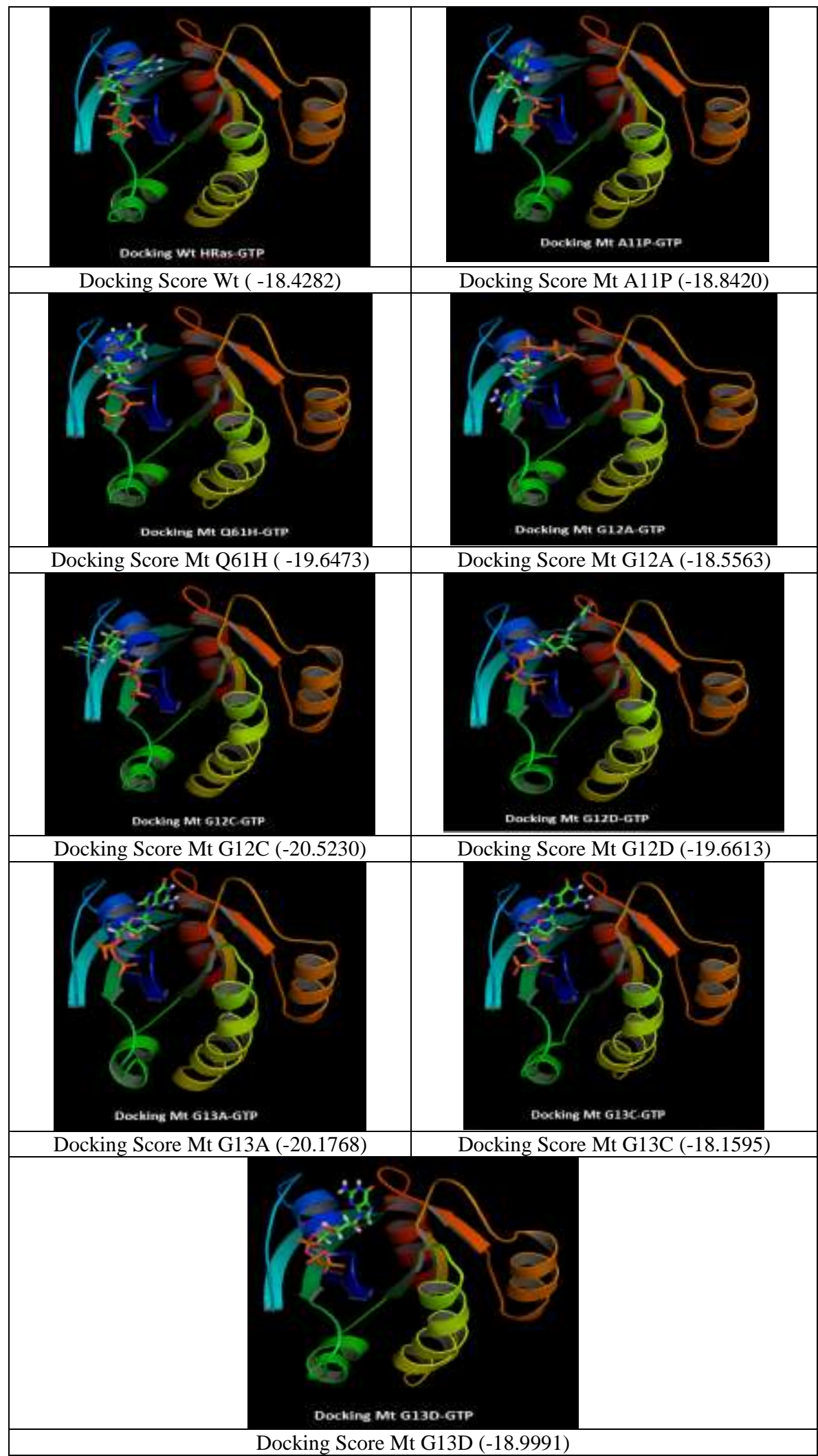

Figure 2. The results of the KRAS Wt and Mt complex with the docking score of GT 
Figure 2 compares the docking results at a specified time interval $(0-200 \mathrm{pc})$ based on the molecular dynamics simulations. Observations were conducted to investigate the potential energy KRAS Mt and Wt before and after the formation of KRAS-GTP complex. The potential energy of KRAS Mt before forming a complex with GTP was significantly higher than KRAS Wt $(\mathrm{p}<0.001)$, except for KRAS Mt-G12D and Q61H that had lower potential energy than KRAS Wt. Further analysis with the Tukey's test revealed a very significant difference $(\mathrm{p}<0.001)$ in KRAS Mt-A11P, G12A, G12C, G12D, and Q61H; in contrast, KRAS Mt-G13A and G13C showed no difference (Table IV).

Table IV. The results of One-way ANOVA of KRAS Wt and all forms of KRAS Mt

\begin{tabular}{|c|c|c|c|c|}
\hline Variables & Means & $95 \% \mathrm{CI}$ & P. Value & $\begin{array}{c}\text { P. Value of the Tukey's } \\
\text { Test }\end{array}$ \\
\hline KRAS Wt & 389.90 & $353.23-426.58$ & & \\
\hline Mt A11P & 496.42 & $458.95-533.88$ & & 0.000 \\
\hline Mt G12A & 552.30 & $515.58-589.03$ & & 0.000 \\
\hline Mt G12C & 501.03 & $461.35-540.72$ & & 0.000 \\
\hline Mt G12D & 298.59 & $260.56-336.63$ & $<0.001$ & 0.001 \\
\hline MtG13A & 436.01 & $397.11-474.90$ & & 0.088 \\
\hline Mt G13C & 424.53 & $389.15-459.92$ & & 0.200 \\
\hline Mt G13D & 520.01 & $483.07-556.95$ & & 0.000 \\
\hline Mt Q61H & 242.31 & $203.06-281.55$ & & 0.000 \\
\hline
\end{tabular}

The potential energy of the proteins after forming a complex with GTP also showed a very significant difference ( $\mathrm{p}<0.001$ ). Increased energy was identified in Mt G12C, G12D, and Q61H. These mutations cause instability in catalytic activity. As reported in Vatanseyer et al. (2017), G12D mutation leads to a shift in the Switch II and a3-helix and this conformational change causes a catalytic disturbance in which the SII movements anti-correlate with other regions. Conversely, decreased potential energy was found in KRAS Wt, Mt A11P, Mt G12A, G13A, G13C, and G13D. Table $\mathbf{V}$ shows a significant difference between the energy before and after the formation of KRASGTP complex ( $p<0.001$ ), except in Mt G12A ( $p>0.01$ ). The mutations in KRAS Mt-A11P, Mt G12A, G13A, G13C, and G13D are suspected of exhibiting similar behavior to KRAS Wt. According to Chen et al. (2013), KRAS mutations at codon 13 and KRAS Wt have identical behavior. Clinical observations on patients whose tumors have either mutant KRAS-Gly12Cys or mutant KRASGly12Val have worse progression-free survival than those with mutant KRAS proteins or wild-type KRAS in their tumors (Ihle et al., 2012). 
Table V. The paired t-test results of the potential energy of KRAS Wt and all KRAS Mt models before and after forming a complex with GTP

\begin{tabular}{lccc}
\hline \multicolumn{1}{c}{ Variables } & Means & P. Value & N \\
\hline KRAS Wt & 389.91 & 0.000 & 201 \\
KRAS Wt-GTP & 292.87 & & \\
Mt A11P & 496.42 & 0.000 & 201 \\
Mt A11P-GTP & 324.20 & & \\
Mt G12A & 552.30 & 0.670 & 201 \\
Mt G12A-GTP & 523.85 & & \\
Mt G12C & 501.03 & 0.000 & 201 \\
Mt G12C-GTP & 701.57 & & \\
Mt G12D & 298.59 & 0.000 & 201 \\
Mt G12D-GTP & 740.24 & & \\
Mt G13A & 436.01 & 0.000 & 201 \\
Mt G13A-GTP & 244.27 & & \\
Mt G13C & 424.53 & 0.000 & 201 \\
Mt G13C-GTP & 355.46 & & \\
Mt G13D & 520.01 & 0.000 & 201 \\
Mt G13D-GTP & 409.51 & & \\
Mt Q61H & 242.31 & 0.000 & 201 \\
Mt Q61H-GTP & 660.09 & & \\
\hline
\end{tabular}

\section{CONCLUSION}

Increased fluctuations in the switch I and switch II regions and the energy after the formation of KRAS-GTP complexes explain why cancer types with mutations at codons 11 and 13 have a better prognosis than the ones at codons 12 and 61.

\section{ACKNOWLEDGMENT}

The authors would like to thank dr Hirowati Ali, Ph.D. and team for their support during this study.

\section{REFERENCES}

Chen, C.C., Er, T.K., Liu, Y.Y., Hwang, J.K., Barrio, M.J., Rodrigo, M., Garcia-Toro, E., Herreros, M., 2013. Computational Analysis of KRAS Mutations: Implications for Different Effects on the KRAS p.G12D and p.G13D Mutations, PLoS ONE, 8(2): e55793.

De Roock W, Claes B, Bernasconi D, De Schutter J, Biesmans B, Fountzilas G, Kalogeras KT, Kotoula V, Papamichael D, Laurent-Puig P, Penault-Llorca F, Rougier P, Vincenzi B, Santini D, Tonini G, Cappuzzo F, Frattini M, Molinari F, Saletti P, De Dosso S, Martini M, Bardelli A, Siena S, Sartore-Bianchi A, Tabernero J, Macarulla T, Di Fiore F, Gangloff AO, Ciardiello F, Pfeiffer P, Qvortrup C, Hansen TP, Van Cutsem E, Piessevaux H, Lambrechts D, Delorenzi M, Tejpar S, 2010. Effects of KRAS, BRAF, NRAS, and PIK3CA mutations on the efficacy of cetuximab plus chemotherapy in chemotherapy-refractory metastatic colorectal cancer: a retrospective consortium analysis, Lancet Oncol, 11(8):753-762.

Forbes, S., Clements, J., Dawson, E., Bamford, S., Webb. T., Dogan, A., Flanagan, A., Teague, J., Wooster, R., Futreal, P.A, 2006. COSMIC 2005. Br J Cancer, 94:318-322.

Forbes, S.A., Bindal, N., Bamford, S., Cole, C., Kok, C.Y., 2011. COSMIC: mining complete cancer genomes in the Catalogue of Somatic Mutations in Cancer Nucleic acids research, 39: 945-950.

Forbes, S.A., Tang, G., Bindal, N., Bamford, S., Dawson, E., Cole, C., Kok, C.Y., Jia, M., Ewing, R., Menzies, A., Teague. J.W., Stratton, M.R., Futreal, P.A., 2009. COSMIC (the Catalogue of 
Somatic Mutations in Cancer): a resource to investigate acquired mutations in human cancer, Nucleic Acids Res, 38: D652-D657.

Futatsugi, N., and Tsuda, M., 2001, Molecular dynamics simulations of Gly-12->Val mutant of p21(ras): dynamic inhibition mechanism, Biophys $J, 81: 3483-3488$.

Gao, C., Leif, A., 2013. Impact of Mutations on KRAS-p120GAP Interaction. Computational Molecular, Bioscience, 3: 9-17.

Garcea, G., Neal, C.P., Pattenden, C.J., 2005. Molecular prognostic markers in pancreatic cancer: A systematic review, Eur J Cancer, 41:2213-2236.

Hardono, B.Y., Santoso, B., and Da'i, M., 2013. Analisis Molecular Docking Energi Ikatan Turunan Diketoperazin (DKP) Sebagai Inhibitor Histon Deasetilasi (HDACi), Master Thesis, Universitas Muhammadiyah Surakarta.

Hongyo, T., Buzard, G.S., Palli, D., Weghorst, C.M., Amorosi, A., Galli, M., 1995. Mutations of the KRAS and p53 genes in gastric adenocarcinomas from a high-incidence region around Florence, Italy. Cancer Res. 55:2665-2672.

Hunter, J.C., Manandhar, A., Carrasco, M.A., Gurbani, D., Gondi, S., and Westover, K.D., 2015. Biochemical and Structural Analysis of Common Cancer-Associated KRAS Mutations, $\mathrm{Mol}$ Cancer Res. 13(9): 1325-1335.

Ihle, N.T., Byers, L.A., Kim, E.S., Saintigny, P., Lee, J.J., Blumenschein, G.R., Tsao, A., Liu, S., Larsen, J.E., Wang, J., Diao, L., Coombes, .R., Chen, L., Zhang, S., Abdelmelek, M.F., Tang,X, Papadimitrakopoulou, V., Minna, J.D., Lippman, S.M., Hong, W.K., Herbst, R.S., Wistuba, I.I., Heymach, J.V., Powis, G., 2012. Effect of KRAS Oncogene Substitutions on Protein Behavior: Implications for Signaling and Clinical Outcome, J Natl Cancer Inst; 104(3): 228-239.

Karnoub, A.E., \& Weinberg, R.A., 2008. RAS oncogenes: split personalities. Nature Reviews Molecular Cell Biology. 9: 517-53.

Knickelbein, K., Zhang, L., 2014. Mutant KRAS as a critical determinant of the therapeutic response of colorectal cancer, Genes and Diseases, 2(1): 4-12.

Krengel, U., Schlichting, I., Scherer, A., Schumann, R., Frech, M., John, J., Kabsch, U., Pai, E.F., Wittinghofer, A., 1990. Three-dimensional structures of HRAS p21 mutants: molecular basis for their inability to function as signal switch molecules, Cell 62(3): 539-548.

Miyakura, Y., Sugano, K., Fukayama, N., Konishi, F., Nagai, H., 2002. Concurrent mutations of KRAS oncogene at codons 12 and 22 in colon cancer. Jpn J Clin Oncol. 32(6):219-21.

Petsko, G.A., Ringe, D., 2004, Protein structure and function. London: New Science Press.

Scheffzek, K., Ahmadian, M.R., Kabsch, W., Wiesmüller, L., Lautwein, A., Schmitz, F., Wittinghofer, A., 1997. The RAS-RASGAP complex: structural basis for GTPase activation and its loss in oncogenic Ras mutants, Science 277 (5324):333-338.

Stephen, A.G., Esposito, D., Bagni, R.K., McCrnick F., 2014. Dragging RAS back in the ring. Cancer Cell, 25:272-281.

Vatansever, S., Erman, B., Gümüş Z.H., 2017. Oncogenic G12D mutation alters local conformations and dynamics of KRAS, bioRxiv preprint first posted online Aug. 19, 2017. 